\title{
BMJ Open Impact of air pollution on renal outcomes: a systematic review and meta- analysis protocol
}

\author{
Aghilès Hamroun (D) , ,,2 Aurore Camier, ${ }^{3}$ Jean Joel Bigna (iD , ${ }^{4}$ François Glowacki ${ }^{1}$
}

To cite: Hamroun A, Camier A, Bigna JJ, et al. Impact of air pollution on renal outcomes: a systematic review and metaanalysis protocol. BMJ Open 2021;11:e041088. doi:10.1136/ bmjopen-2020-041088

\section{- Prepublication history and} additional material for this paper are available online. To view these files, please visit the journal online (http://dx.doi org/10.1136/bmjopen-2020041088).

Received 02 June 2020 Revised 02 December 2020 Accepted 05 January 2021

Check for updates

(c) Author(s) (or their employer(s)) 2021. Re-use permitted under CC BY-NC. No commercial re-use. See rights and permissions. Published by BMJ.

${ }^{1}$ Nephrology, Regional and University Hospital Centre Lille, Lille, France

${ }^{2}$ Clinical Epidemiology Team, INSERM U1018, Villejuif, France ${ }^{3}$ Research Team on Early Life Origins of Health (EAROH), UMR1153 Centre of Research in Epidemiology and Statistics (CRESS), Paris, France ${ }^{4}$ Department of Epidemiology and Public Health, Centre Pasteur of Cameroon, Yaoundé, Cameroon

Correspondence to

Dr Aghilès Hamroun; aghiles.hamroun@inserm.fr

\section{ABSTRACT}

Introduction Chronic kidney disease is a serious and a frequent disease associated with a high risk of morbimortality. Although several risk factors have already been well addressed, mostly diabetes and hypertension, many remain underappreciated, such as chronic exposure to air pollution.

Methods and analysis We will search EMBASE, PubMed, Web of Science, Cochrane Library and CINAHL database, from inception to 31 March 2020, for relevant records using a combination of keywords related to the type of exposure (ozone, carbon monoxide, nitrogen oxides and dioxide, sulfur dioxide, $\mathrm{PM}_{2.5}, \mathrm{PM}_{\text {coarse }}$ and $\mathrm{PM}_{10}$ ) and to the type of outcome (chronic kidney disease, endstage renal/kidney disease, kidney failure, proteinuria/ albuminuria, renal function, renal transplant, kidney graft, kidney transplant failure, nephrotic syndrome and kidney cancer). The review will be reported according to the guidelines of the Meta-analysis Of Observational Studies in Epidemiology. Two independent reviewers will select studies without design or language restrictions, using original data and investigating the association between exposure to one or more of the prespecified air pollutants and subsequent risk of renal outcomes. Using randomeffects meta-analyses, we will present pooled summary statistics (HR, OR or beta-coefficients with their respective $95 \% \mathrm{Cl}$ ) associated with a standardised increase in each pollutant level. The results will be presented by air pollutant and outcome. Heterogeneity will be assessed using the $\chi^{2}$ test on Cochran's $Q$ statistic and quantified by calculating $1^{2}$. The Egger's test and visual inspection of funnel plots will be used to assess publication bias.

Ethics and dissemination Since primary data are not collected in this study, ethical approval is not required. This review is expected to provide relevant data on the associations between various air pollutants' exposure and renal outcomes. The final report will be published in an international peer-reviewed journal.

PROSPERO registration number CRD42020187956.

\section{INTRODUCTION}

Chronic kidney disease (CKD), defined according to the KDIGO (Kidney Disease: Improving Global Outcomes) as at least 3 months of either reduced glomerular filtration rate $\left(<60 \mathrm{~mL} / \mathrm{min} / 1.73 \mathrm{~m}^{2}\right)$ or evidence of kidney damage such as albuminuria or abnormal pathology, ${ }^{12}$ affects around $10 \%$

\section{Strengths and limitations of this study}

This will be the first exhaustive systematic review summarising data on the association between various types of air pollutants and multiple renal outcomes, including chronic kidney disease, end-stage renal disease, renal function, proteinuria, nephrotic syndrome, kidney graft failure and kidney cancer.

- Rigorous methods and robust statistical analysis will be used to minimise bias and provide accurate data.

- No study design or language restriction will be applied, hence allowing inclusion of the maximum number of studies in this review.

- Multiple sources of heterogeneity between studies (regarding nature and assessment method for air pollutant exposure, study design and population characteristics) may represent an important shortcoming.

of the world's population. ${ }^{34}$ This represents a serious illness, fraught with a significant risk of cardiovascular events, hospitalisations and mortality, ${ }^{5}$ generating at the same time significant costs to the society. Mortality attributed to CKD worldwide is constantly increasing, the Global Burden of Disease having noted an up to $30 \%$ inflation since 2005 . ${ }^{6}$ The main risk factors for this pathology are represented by diabetes and hypertension, and constitute almost $50 \%$ of the responsible aetiologies. ${ }^{7-10}$ Nevertheless, there seems to be a discrepancy between the evolving trends of these risk factors and the steadily increasing global burden of CKD, suggesting that there are other unexplored causes contributing to the increase of the disease worldwide. ${ }^{11-13}$

Among these unappreciated factors, environmental factors seem to have drawn more and more attention in the last decade, ${ }^{12} 14-17$ air pollution having even been recognised as one of the leading causes of global disease burden. ${ }^{18} 19$ Since the discovery of kidney damage induced by the inhalation of diesel in rodent models, the association between air pollution and renal outcomes has become a trendy area of research. ${ }^{20-22}$ Air pollution 
corresponds to a complex mixture of gaseous components and air-suspended solid/liquid particles, due to a large variety of sources: from particulate matter mostly trafficrelated, to gaseous pollutants such as nitrogen oxides, sulfur oxides, ozone and carbon monoxide issued from industrial production and road traffic. ${ }^{1523-26}$ Although there are a few epidemiological data on the association between these pollutants and renal outcomes, these data remain very scarce. ${ }^{141617}$ Moreover, the two recent reviews have mainly focused on the link between CKD incidence and chronic exposure to particulate matter. ${ }^{16}{ }^{17}$ Multiple sources of methodological disparities have been noticed between these studies, with regard to the nature and the assessment method of exposure, as well as the type and definition of the studied outcomes, making any synthesis really challenging.

Through this systematic review and meta-analysis, we will attempt to exhaustively summarise the current evidence on the association between main air pollutant's exposure and various renal outcomes, including CKD, renal function biomarkers, kidney transplant failure and renal parenchyma neoplasm.

\section{Review question}

What is the impact of common air pollutants on various renal outcomes?

\section{Objectives}

This systematic review and meta-analysis aims at determining the association between various types of air pollutants $\left(\mathrm{PM}_{2.5}, \mathrm{PM}_{10}, \mathrm{PM}_{\text {coarse }}\right.$, Nitrite Oxide $\left(\mathrm{NO}_{\mathrm{x}}\right)$, Nitrite Dioxide $\left(\mathrm{NO}_{2}\right)$, Sulfur Dioxide $\left(\mathrm{SO}_{2}\right)$, Ozone $\left(\mathrm{O}_{3}\right)$, Carbon oxide $(\mathrm{CO}))$ and the following:

- Risk of CKD (prevalence and incidence).

- Risk of end-stage renal disease (ESRD).

- Renal function decline (based on estimated glomerular filtration rate, eGFR).

- Risk of proteinuria/albuminuria development.

- Risk of nephrotic syndrome.

- Risk of kidney transplant failure.

- Risk of kidney cancer.

\section{METHODS AND ANALYSIS}

This systematic review and meta-analysis will be reported in conformity with the guidelines of the Meta-analysis Of Observational Studies in Epidemiology. ${ }^{27}$ The Preferred Reporting Items for Systematic Review and Meta-Analysis Protocols (PRISMA-P) was used to report this protocol. ${ }^{28}$ The PRISMA-P checklist is attached as online supplemental file 1 .

\section{Criteria for considering studies for the review}

- Population: all studies in human beings which present original data and are published in full text or meeting abstract will be eligible for inclusion, with no restrictions on study design, publication date, language or ethnicity. We will exclude animal studies, ex vivo and toxicological studies, commentaries and editorials, case reports, and studies with no original data.
If a citation lacks enough quantitative data and these essential data cannot be obtained from the corresponding author, the study will be excluded.

- Exposure will be defined as any method of air pollutant exposure measurement, including assessments of pollutant concentration by monitoring stations, use of satellite-based or land-use regression models, and use of indicators of long-term traffic exposure..$^{25}$ 29-31

- Comparator: Because air pollutant exposure is often presented as a continuous level regarding a specific population study, there will not be any comparator group. The generated effect will be expressed for an appropriate standardised increase of air pollutant exposure (eg, per $10 \mu \mathrm{g} / \mathrm{m}^{3}$ increase of $\mathrm{PM}_{2.5}$ exposure level).

- Renal outcomes, including CKD, ESRD, proteinuria, renal function, nephrotic syndrome, kidney graft failure and kidney cancer, will be defined on respective International Classification of Diseases (ICD) diagnosis codes (detailed list in online supplemental file 2$)^{32}$ or clinically confirmed diagnosis. CKD will be mainly defined as an eGFR $<60 \mathrm{~mL} / \mathrm{min} / 1.73 \mathrm{~m}^{2}$ for at least 3 months, ESRD as the need for renal replacement therapy (dialysis or kidney transplantation), proteinuria as urinary protein level $>0.5 \mathrm{~g} / 24$ hours or urinary protein to creatinine ratio $>0.5 / \mathrm{g}$, albuminuria as urinary albumin level $>30 \mathrm{mg} / 24$ hours or urinary albumin to creatinine ratio $>30 \mathrm{mg} / \mathrm{g}$, and kidney transplant failure as the need to return on renal replacement therapy after kidney transplantation. ${ }^{1}$

\section{Search strategy for identifying relevant studies}

The search strategy will be conducted as follows.

\section{Bibliographic database searches}

Relevant records will be identified by searching EMBASE, PubMed, Web of Science, Cochrane Library and CINAHL database, from inception to 31 March 2020. We will use a combination of keywords related to the type of exposure ('air pollution', 'air pollutants', 'ozone', 'carbon monoxide', 'sulfur dioxide', 'nitrogen dioxide', 'particulate matter', ' $\mathrm{PM}_{2.5}$ ', 'PM $\mathrm{PMarse}_{\text {' }}$ and ' $\mathrm{PM}_{10}$ ') and to the type of outcome ('chronic kidney disease', 'end-stage renal/kidney disease', 'kidney failure', 'renal function', 'proteinuria', 'albuminuria', 'renal transplant', 'kidney graft', 'kidney graft failure', 'nephrotic syndrome' and 'kidney cancer'). Online supplemental file 3 shows the full search strategy for EMBASE that will be adapted to fit with other databases. No language restriction will be applied. For articles published in a language other than English and French, an experienced translator in the concerned language will be contacted for translation.

\section{Searching for other sources}

We will scan the references of all relevant articles for additional relevant data sources missed during our search and their full texts will be retrieved. References of pertinent reviews will also be scanned. 
Selection of studies for inclusion in the review

All references identified after implementation of the search strategy will be imported to the Zotero software. All records obtained from various databases will be combined in a single Zotero library and duplicates will be removed. Two reviewers ( $\mathrm{AH}$ and $\mathrm{AC}$ ) will independently evaluate the studies obtained from the searches using an assessment form to ensure that the selection criteria are reliably applied. These reviewers will screen the titles and abstracts of the papers obtained, after which the full texts of potentially eligible papers will be retrieved by one reviewer $(\mathrm{AH})$. The two reviewers will independently review the full text of each potentially eligible study, compare their results and resolve any discrepancy by discussion. For duplicates, or studies published in more than one report, the one reporting the largest sample size will be considered. Studies with inaccessible full text either online or from the corresponding author will be excluded.

\section{Assessment of methodological quality and reporting of data}

The Newcastle-Ottawa Scale, with some modifications, has been adapted to judge study quality, according to validated scales in previous studies and the Cochrane Collaboration. ${ }^{33-35}$ Two independent reviewers will assess each study for potential biases on four major components, namely exposure assessment bias, detection bias, selection bias and adjustment for confounders. For exposure assessment bias, we will deem studies using less than three fixed-site monitors to assign participant air pollutant exposure levels as having a high risk of exposure assessment bias, studies using three or more as having moderate risk, and studies using personal exposure or atmospheric modelling as having low risk. We will also regard studies as having a high risk of exposure assessment bias if they were done before 1980 because insufficient technological and methodological precision in measuring and assigning particulate matter exposure was available during that period. We will deem studies with health outcomes not based on ICD-10 diagnosis codes or clinically confirmed outcomes to have a high risk of detection bias and studies with unrepresentative study populations to have a high risk of selection bias. We will also regard studies that did not adjust for at least three of the following main confounders-long-term trends, seasonality, weather, population characteristics and lifestyle factors (such as smoking status, diabetes or body mass index)—as having a high risk of bias. After considering all four domains in the overall assessment, the risk of bias will be classified as 'low', 'moderate', 'high' or 'uncertain'. The detailed criteria for the methodological quality assessment are summarised in online supplemental file 4 .

\section{Data extraction and management}

A data extraction form will be used to collect information on the surname of the first author, year of publication, country where the study was conducted, study design, study area (rural, urban), research period, sampling method, timing of data collection, population setting (general population, hospitalised patients), nature and definition of studied outcomes, assessment method of air pollutant exposure, mean or median age, proportion of men, ethnicity, other specific characteristics of the study population (such as proportions of active smoking, diabetes, obesity or cardiovascular comorbidities), sample size, crude and adjusted estimates (OR, HR and beta-coefficients) for the association between each air pollutant and the prespecified outcomes of interest, as well as adjustment factors included in multivariate analyses. We will exclude studies in which relevant data are impossible to extract even after contacting the corresponding author.

\section{Data synthesis and analysis}

Due to the expected heterogeneity in both pollutant and population characteristics resulting from differing study designs, we will pool crude and adjusted estimates using the random-effects method of DerSimonian and Laird, incorporating both between-study and within-study variation. ${ }^{36}$ We will present pooled summary statistics as the risk ratio (OR or HR, when appropriate) (binary outcomes) or beta-coefficient (continuous outcome) associated with a standardised increase in air pollutant levels, assuming that most studies have verified the linearity assumption. ${ }^{37}$ We will choose the levels of exposure that will be used most frequently for each studied pollutant. If the increment in pollutant concentration is not equivalent to the chosen one, standardised risk estimates will be calculated using the following formula: $\mathrm{OR}_{\text {(standardised) }}=\mathrm{OR}^{\text {increment }}$ (10)/increment (original) (the same formula will be applied to standardise HRs and beta-coefficients). All pooled estimates will be pooled by pollutant and renal outcome and reported with $95 \%$ CI. Given the potential study design heterogeneity, the results will be stratified by the nature of included studies: on the one hand the results from crosssectional studies and on the other hand those from longitudinal studies. Regarding CKD outcome, for example, the results will be presented according to whether these are prevalence (summarised as OR $(95 \% \mathrm{CI})$ ) or incidence data (summarised as HR $(95 \% \mathrm{CI})$ ). One study estimate will be included per city for the same study period to ensure results will not be biased by multiple inclusions of one city data. Where duplicate cities will be presented for the same study period, we will select one estimate for meta-analysis by prioritising multicity designs because of their standardised and often higher quality methodologies, and then if duplicates are still present selecting the study with the lower risk of exposure assessment bias. As a sensitivity analysis, we will also pool the fully adjusted estimates using a fixed-effects method.

Heterogeneity will be assessed using the $\chi^{2}$ test on Cochran's $Q$ statistic and quantified by calculating $\mathrm{I}^{2} .{ }^{38} \mathrm{I}^{2}$ values of $25 \%, 50 \%$ and $75 \%$ will, respectively, represent low, medium and high heterogeneity. We will assess the presence of publication bias using funnel plots inspection (if $\geq 10$ studies) and Egger's test (if $\geq 3$ studies). ${ }^{39}$ When 
they will be enough data, meta-regression and subgroup analyses will be performed to investigate any possible sources of heterogeneity using the aforementioned variables and the study quality. In case of substantial clinical heterogeneity or insufficient data, a narrative summary of findings will also be done. Counterenhanced funnel plot (if $\geq 10$ studies) and Harbord test (if $\geq 3$ studies) will be used to assess the presence of publication bias. ${ }^{40}$

The inter-rater agreement for study inclusion between investigators will be assessed using Cohen's $\kappa$ coefficient. ${ }^{41}$ Data analyses will be done using the 'meta' package of the R V.3.6.2 statistical software.

\section{Presentation and reporting of results}

The study selection process will be summarised using a flow diagram. Quantitative data will be presented in tables of individual studies and in summary tables, or forest plots where appropriate. The quality scores of bias for each eligible study will be reported accordingly.

\section{Patient and public involvement}

Patients and the public will not be involved in the design or planning of the study.

\section{Potential amendments}

We do not plan to modify the protocol to avoid reporting bias. However, if necessary, any amendment in the review process will be reported for transparency.

\section{Ethics and dissemination}

Since primary data will not be collected in this study, ethical approval is not required. This review is expected to provide accurate data on the association between air pollution and various renal outcomes. The final report will be published in an international peer-reviewed journal.

\section{Review status}

Preliminary searches.

Contributors $\mathrm{AH}$ and $\mathrm{FG}$ had the idea. $\mathrm{AH}, \mathrm{FG}, \mathrm{AC}$ and $\mathrm{JJB}$ designed and conceived the protocol. AH drafted the manuscript. $A H, A C, J J B$ and $F G$ critically revised the manuscript for methodology and intellectual content. $\mathrm{AH}$ and $\mathrm{AC}$ are the guarantors of the review. All authors approved the final version of this manuscript.

Funding The authors have not declared a specific grant for this research from any funding agency in the public, commercial or not-for-profit sectors.

Competing interests None declared.

Patient consent for publication Not required.

Provenance and peer review Not commissioned; externally peer reviewed.

Supplemental material This content has been supplied by the author(s). It has not been vetted by BMJ Publishing Group Limited (BMJ) and may not have been peer-reviewed. Any opinions or recommendations discussed are solely those of the author(s) and are not endorsed by BMJ. BMJ disclaims all liability and responsibility arising from any reliance placed on the content. Where the content includes any translated material, BMJ does not warrant the accuracy and reliability of the translations (including but not limited to local regulations, clinical guidelines, terminology, drug names and drug dosages), and is not responsible for any error and/or omissions arising from translation and adaptation or otherwise.

Open access This is an open access article distributed in accordance with the Creative Commons Attribution Non Commercial (CC BY-NC 4.0) license, which permits others to distribute, remix, adapt, build upon this work non-commercially, and license their derivative works on different terms, provided the original work is properly cited, appropriate credit is given, any changes made indicated, and the use is non-commercial. See: http://creativecommons.org/licenses/by-nc/4.0/.

\section{ORCID iDs}

Aghilès Hamroun http://orcid.org/0000-0003-4988-206X

Jean Joel Bigna http://orcid.org/0000-0001-8018-6279

\section{REFERENCES}

1 Levin A, Stevens PE, Bilous RW. Kidney disease: improving global outcomes (KDIGO) CKD work group. KDIGO 2012 clinical practice guideline for the evaluation and management of chronic kidney disease. Kidney International Supplements 2013;3:1-150.

2 Eckardt K-U, Kasiske BL. Kidney disease: improving global outcomes. Nat Rev Nephrol 2009;5:650-7.

3 Mills KT, Xu Y, Zhang W, et al. A systematic analysis of worldwide population-based data on the global burden of chronic kidney disease in 2010. Kidney Int 2015;88:950-7.

4 Coresh J, Selvin E, Stevens LA, et al. Prevalence of chronic kidney disease in the United States. JAMA 2007;298:2038-47.

5 Go AS, Chertow GM, Fan D, et al. Chronic kidney disease and the risks of death, cardiovascular events, and hospitalization. $N$ Engl J Med 2004;351:1296-305.

6 GBD 2015 Mortality and Causes of Death Collaborators. Global, regional, and national life expectancy, all-cause mortality, and causespecific mortality for 249 causes of death, 1980-2015: a systematic analysis for the global burden of disease study 2015. Lancet 2016;388:1459-544.

7 Lea JP, Nicholas SB. Diabetes mellitus and hypertension: key risk factors for kidney disease. J Natl Med Assoc 2002;94:7S-15.

8 Perneger TV, Brancati FL, Whelton PK, et al. End-Stage renal disease attributable to diabetes mellitus. Ann Intern Med 1994;121:912-8.

9 Atkins RC. The epidemiology of chronic kidney disease. Kidney Int 2005;67:S14-18.

10 Saran R, Robinson B, Abbott KC, et al. Us renal data system 2019 annual data report: epidemiology of kidney disease in the United States. Am J Kidney Dis 2020;75:A6-7.

11 Brück K, Stel VS, Gambaro G, et al. Ckd prevalence varies across the European general population. J Am Soc Nephrol 2016;27:2135-47.

$12 \mathrm{Xu} \mathrm{X}$, Nie S, Ding H, et al. Environmental pollution and kidney diseases. Nat Rev Nephrol 2018;14:313-24.

13 Grams ME, Juraschek SP, Selvin E, et al. Trends in the prevalence of reduced GFR in the United States: a comparison of creatinine- and cystatin C-based estimates. Am J Kidney Dis 2013;62:253-60.

14 Al-Aly Z, Bowe B. Air pollution and kidney disease. Clin J Am Soc Nephrol 2020;15:301-3.

15 Afsar B, Elsurer Afsar R, Kanbay A, et al. Air pollution and kidney disease: review of current evidence. Clin Kidney J 2019;12:19-32.

16 Wu M-Y, Lo W-C, Chao C-T, et al. Association between air pollutants and development of chronic kidney disease: a systematic review and meta-analysis. Sci Total Environ 2020;706:135522.

17 Liu B, Fan D, Huang F. Relationship of chronic kidney disease with major air pollutants - A systematic review and meta-analysis of observational studies. Environ Toxicol Pharmacol 2020;76:103355.

18 Brook RD, Newby DE, Rajagopalan S. The global threat of outdoor ambient air pollution to cardiovascular health: time for intervention. JAMA Cardiol 2017;2:353-4.

19 GBD 2015 Risk Factors Collaborators. Global, regional, and national comparative risk assessment of 79 behavioural, environmental and occupational, and metabolic risks or clusters of risks, 1990-2015: a systematic analysis for the global burden of disease study 2015 . Lancet 2016;388:1659-724.

20 Nemmar A, Karaca T, Beegam S, et al. Prolonged pulmonary exposure to diesel exhaust particles exacerbates renal oxidative stress, inflammation and DNA damage in mice with adenine-induced chronic renal failure. Cell Physiol Biochem 2016;38:1703-13.

21 Miller MR, Raftis JB, Langrish JP, et al. Inhaled nanoparticles accumulate at sites of vascular disease. ACS Nano 2017;11:4542-52.

22 Tavera Busso I, Mateos AC, Juncos LI, et al. Kidney damage induced by sub-chronic fine particulate matter exposure. Environ Int 2018;121:635-42.

23 Kellogg WW, Cadle RD, Allen ER, et al. The sulfur cycle. Science 1972;175:587-96.

24 Chen S-Y, Chu D-C, Lee J-H, et al. Traffic-Related air pollution associated with chronic kidney disease among elderly residents in Taipei City. Environ Pollut 2018;234:838-45. 
25 Kelly FJ, Fuller GW, Walton HA, et al. Monitoring air pollution: use of early warning systems for public health. Respirology 2012;17:7-19.

26 Luecken DJ, Napelenok SL, Strum M, et al. Sensitivity of ambient atmospheric formaldehyde and ozone to precursor species and source types across the United States. Environ Sci Technol 2018;52:4668-75.

27 Stroup DF, Berlin JA, Morton SC, et al. Meta-Analysis of observational studies in epidemiology: a proposal for reporting. meta-analysis of observational studies in epidemiology (moose) group. JAMA 2000;283:2008-12.

28 Moher D, Shamseer L, Clarke M, et al. Preferred reporting items for systematic review and meta-analysis protocols (PRISMA-P) 2015 statement. Syst Rev 2015;4:1.

29 Hidy GM, Brook JR, Chow JC, et al. Remote sensing of particulate pollution from space: have we reached the promised land? J Air Waste Manag Assoc 2009;59:1130-9.

30 Sorek-Hamer M, Just AC, Kloog I. Satellite remote sensing in epidemiological studies. Curr Opin Pediatr 2016;28:228-34.

31 Ryan $\mathrm{PH}$, LeMasters GK. A review of land-use regression models for characterizing intraurban air pollution exposure. Inhal Toxicol 2007;19 Suppl 1:127-33.

32 Steindel SJ. International classification of diseases, 10th edition, clinical modification and procedure coding system: descriptive overview of the next generation HIPAA code sets. J Am Med Inform Assoc 2010;17:274-82.
33 Tang L, Wang Q-Y, Cheng Z-P, et al. Air pollution and venous thrombosis: a meta-analysis. Sci Rep 2016;6:32794.

34 Mustafic $\mathrm{H}$, Jabre P, Caussin C, et al. Main air pollutants and myocardial infarction: a systematic review and meta-analysis. JAMA 2012;307:713-21.

35 Shah ASV, Langrish JP, Nair $\mathrm{H}$, et al. Global association of air pollution and heart failure: a systematic review and meta-analysis. Lancet 2013;382:1039-48.

36 DerSimonian R, Laird N. Meta-Analysis in clinical trials revisited. Contemp Clin Trials 2015;45:139-45.

37 Peña EA, Slate EH. Global validation of linear model assumptions. $J$ Am Stat Assoc 2006;101:341-54.

38 Huedo-Medina TB, Sánchez-Meca J, Marín-Martínez F, et al. Assessing heterogeneity in meta-analysis: Q statistic or 12 index? Psychol Methods 2006;11:193-206.

39 Egger M, Smith GD, Schneider M, et al. Bias in meta-analysis detected by a simple, graphical test. BMJ 1997;315:629-34.

40 Harbord RM, Egger M, Sterne JAC. A modified test for small-study effects in meta-analyses of controlled trials with binary endpoints. Stat Med 2006;25:3443-57.

41 McHugh ML. Interrater reliability: the kappa statistic. Biochem Med 2012;22:276-82. 